\title{
Tone Burst Masseter Vestibular Evoked Myogenic Potentials: Normative Values and Test-Retest Reliability
}

\author{
Sangu Srinivasan Vignesh, MSc (Audiology) ${ }^{1} \quad$ Niraj Kumar Singh, PhD (Audiology) ${ }^{2}$ \\ Krishna Rajalakshmi, PhD (Speech \& Hearing) ${ }^{2}$ \\ ${ }^{1}$ Department of Neurology, Tamil Nadu Government Multi Super \\ Speciality Hospital, Chennai, Tamil Nadu, India \\ ${ }^{2}$ Department of Audiology, All India Institute of Speech and Hearing, \\ Address for correspondence Sangu Srinivasan Vignesh, \\ vigneshaslp@gmail.com \\ Mysuru, Karnataka, India \\ J Am Acad Audiol 2021;32:308-314.
}

\begin{abstract}
Background Masseter vestibular evoked myogenic potential (mVEMP) is a recent tool for the assessment of vestibular and trigeminal pathways. Though a few studies have recorded mVEMP using click stimuli, there are no reports of these potentials using the more conventional VEMP eliciting stimuli, the tone bursts.

Purpose The aim of the study is to establish normative values and determine the testretest reliability of tone burst evoked mVEMP.

Research Design The research design type is normative study design.

Study Sample Forty-four healthy participants without hearing and vestibular deficits in the age range of 18 to 50 years participated in the study.

Data Collection and Analysis All participants underwent mVEMP testing using $500 \mathrm{~Hz}$ tone-burst stimuli at $125 \mathrm{~dB}$ peSPL. Ten participants underwent second mVEMP testing within 1 month of the initial testing to estimate the test-retest reliability.

Results Tone burst mVEMP showed robust responses in all participants. There were no significant ear and sex differences on any mVEMP parameter $(p>0.05)$; however, males had significantly higher EMG normalized peak-to-peak amplitude than females. Intraclass correlation coefficient (ICC) values of tone burst mVEMP showed excellent test-retest reliability (ICC $>0.75$ ) for ipsilateral and contralateral p11 latency, ipsilat-

Keywords

- vestibulomasseteric reflex

- evoked potentials

- otolith responses

- trigeminal nerve eral EMG normalized p11-n21 peak to peak amplitude, and amplitude asymmetry ratio. Fair and good test-retest reliability $(0.4<\mathrm{ICC}>0.75)$ was observed for ipsilateral and contralateral n21 latency, contralateral EMG normalized peak-to-peak amplitude, and amplitude asymmetry ratio.

Conclusion Tone burst mVEMP is a robust and reliable test for evaluating the functional integrity of the vestibulomasseteric reflex pathway.
\end{abstract}

Vestibular evoked myogenic potentials (VEMPs) are otolithic responses induced by sound, vibration, or galvanic stimulation. ${ }^{1}$ Two clinical variants are popularly used, one recorded from the sternocleidomastoid muscle, known as cervical VEMP (cVEMP), and the other from the inferior oblique extraocular muscle, called ocular VEMP (oVEMP). Practically, VEMP

received

September 18, 2020

accepted after revision

November 30, 2020

published online

June 1, 2021

can also be recorded from other muscles of the body including the gastrocnemius muscle, ${ }^{2}$ the triceps muscle, ${ }^{3}$ the trapezius muscles, ${ }^{4}$ and the masseter muscle. ${ }^{5}$ The responses from the masseter muscle are called the masseter vestibular evoked myogenic potential (mVEMP) ${ }^{6}$ represented as bilaterally symmetrical biphasic $\mathrm{p} 11-\mathrm{n} 21$ responses. $^{5}$

(C) 2021. American Academy of Audiology. All rights reserved. Thieme Medical Publishers, Inc., 333 Seventh Avenue, 18th Floor, New York, NY 10001, USA
DOI https://doi.org/ 10.1055/s-0041-1728718. ISSN 1050-0545. 
mVEMP was originally studied by Deriu and colleagues ${ }^{5,7-9}$ and it consists of two components. ${ }^{5}$ They are: (1) vestibular origin-short-latency, higher threshold (90-100 dBnHL) p11-n15 wave and, (2) auditory origin-longer latency, low threshold ( $<80 / 90 \mathrm{dBnHL}$ ) p16-n21 wave. In healthy humans, $\mathrm{n} 15$ response is reported to appear as a small deflection in the biphasic response and is often undetectable. Though the vestibular end organ for the generation of mVEMP is possibly saccule as in cVEMP, ${ }^{5,8}$ it lacks experimental evidence. mVEMP is reported to be useful in central vestibular disorders, especially multiple sclerosis ${ }^{10,11}$ and Parkinson's disease. ${ }^{6,12}$

mVEMP is a relatively new tool and the normative data are available for only click stimuli. ${ }^{13,14}$ However, the literature on cVEMP and oVEMP has shown that they are more robust when elicited by $500 \mathrm{~Hz}$ tone bursts than the clicks. ${ }^{15-18}$ Also study on frequency tuning of cVEMP and oVEMP ${ }^{19}$ shows that the lowest VEMP threshold is obtained at $500 \mathrm{~Hz}$. Further, as mVEMP eventually becomes widely recognized test for saccular function, it is likely that it would be used for the assessment of saccular function and the functional integrity of the vestibulotrigeminal neural pathways. However, test-retest reliability is the most important aspect of any test, the data on which is missing from the literature, especially for the $500-\mathrm{Hz}$ tone burst-induced mVEMP. Therefore, the present study aimed to establish the normative values for tone burst mVEMP and determine its test-retest reliability.

\section{Methods}

\section{Participants}

Forty-four healthy individuals (12 males and 32 females) with a mean age of 31.49 years (range 18-50 years) were included in the study. None of the participants had a history of auditory disorders, vestibular disorders, and systemic diseases. Otoscopic examination showed normal external ear and tympanic membrane. Pure tone audiometry showed hearing threshold within normal limits (pure-tone average $\leq 15 \mathrm{dBHL}$ ). They all had normal oromandibular structure. Ten participants (three males and seven females) from the abovementioned healthy individuals underwent a second testing session within 1 month of the initial testing. Ethical committee approval was obtained from the ethical committee for biobehavioral research of the institute (DOR.9.1/ph.d/ PBJ/1071/2015-16). All the participants gave written informed consent for the mVEMP testing.

\section{Recording of $\mathbf{m V E M P}$}

mVEMP testing was performed using a commercial dualchannel diagnostic evoked potential instrument (Neurosoft, Russia) in a sound-treated room. The participants were seated in a comfortable chair with an upright posture. mVEMP was elicited by tone bursts of $500 \mathrm{~Hz}$ (2-0-2 cycle) delivered at $125 \mathrm{~dB}$ peSPL in compliance with the safety recommendations by Singh et al. ${ }^{20}$ Stimuli were presented monaurally at the rate of $5.1 \mathrm{~Hz}$ into the ear canal through the etymotic ER3A insert earphones. The surface electromyographic (EMG) activity of masseter muscle was picked-up using the belly tendon configuration with an active electrode placed on the lower third of

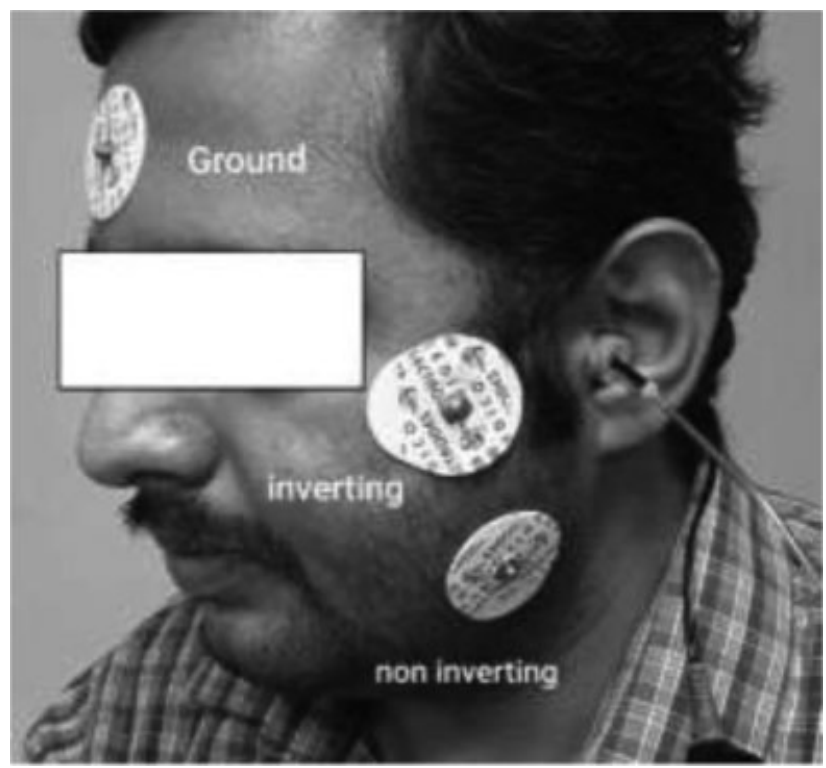

Fig. 1 Electrode placement for recording masseter VEMP. The active electrode is positioned on the lower third of the masseter muscle, the reference electrode on the zygomatic arch, and ground on the forehead. VEMP, vestibular evoked myogenic potential.

the masseter muscle, reference electrode over the zygomatic arch ( $2 \mathrm{~cm}$ above the active electrode), and ground over the forehead $^{5}$ as seen in - Fig. 1. Absolute and interelectrode impedance was maintained below 5 and $2 \mathrm{k} \Omega$, respectively. The two-channel recording was used to record ipsilateral and contralateral responses from both masseter muscles and each response was recorded twice to ensure the reproducibility. The in-built visual display of the EMG needle deflection was used to give visual feedback on the computer screen. Maximum voluntary contraction of the masseter muscle was calculated by measuring the maximum EMG needle deflection during the forceful bite of the jaw before initiation of recordings. From the maximum voluntary contraction values, the target level to maintain masseter muscle tension was set between 30 and 50\% and the participants were asked to maintain muscle tension within these target levels during recording. The mean and standard deviation values for maximum voluntary contraction were 180.18 and $50.94 \mu \mathrm{V}$, respectively. A rest period of approximately 2 minutes was given after each recording to avoid fatigue. The mVEMP responses were band-pass filtered from 0.3 to $2,000 \mathrm{~Hz}$, amplified by a factor of 5,000 and averaged for 300 stimuli. Each response was recorded with a time window of 150 milliseconds ( $50 \mathrm{~ms}$ before and $100 \mathrm{~ms}$ after stimulus delivery). After recording, EMG normalization was used to counter the differential effects of muscle tension over the mVEMP amplitude within and across the participants.

\section{Response Analyses}

Each waveform of mVEMP was analyzed by two experienced audiologists independently to identify peaks. Peak latencies of p11 and n21, EMG normalized peak-to-peak amplitude (from p11 to n21), and mean EMG were obtained for each waveform. EMG normalized amplitudes are expressed as the ratio between the absolute peak-to-peak amplitude of the response 
in the post-stimulus time frame and the mean root mean square of EMG activity in the prestimulus time period. ${ }^{5}$ Amplitude asymmetry ratio was obtained using the Jongkee's formula (shown in the equation below) which is also used for calculating the amplitude asymmetry ratio of cVEMP and oVEMP. ${ }^{21}$ Amplitude asymmetries were calculated for ipsilateral and contralateral waveforms separately.

\section{Amplitude asymmetry ratio $=$} $\frac{\text { Right ear EMG normalized amplitude-Left ear EMG normalized amplitude }}{\text { Right ear EMG normalized amplitude+Left ear EMG normalized amplitude }} \times 100$

\section{Statistical Analysis}

Statistical analyses were performed using the statistical package for the social sciences (SPSS) software version 20. The Shapiro-Wilk's test of normality showed that the data followed non-normal distribution $(p<0.05)$ for all parameters of the tone burst mVEMP. Therefore, nonparametric statistical tests were used throughout. The Wilcoxon signed ranks test was used for between the ear comparisons. The Mann-Whitney $U$ test was used for between-groups comparisons for evaluating the sex differences. The test-retest reliability was assessed by the intraclass correlation coefficient (ICC). ICC values were categorized in the way Versino et $\mathrm{al}^{22}$ categorized them for labeling the test-retest reliability of cVEMP. The ICC values above 0.75 represent excellent reliability, values between 0.4 and 0.75 represent fair-to-good reliability, and values below 0.4 represent poor test-retest reliability.

\section{Results}

\section{Normative Values of Tone Burst mVEMP}

The mean, median values, and standard deviation of p11 latency, n21 latency, EMG normalized peak-to-peak amplitude, and mean rectified EMG of ipsilateral and contralateral tone burst mVEMP responses recorded from right and left ears are shown in - Table 1. All 44 healthy participants had p11 and n21 peaks in both the ears. Both these peaks were consistently present and clear among all individuals. The right and left ear comparison was performed using the Wilcoxon signed-rank test. The Wilcoxson signed-rank test showed no significant difference between the ears on ipsilateral mVEMP parameters including ipsilateral p11 latency $(Z=4.08, p=0.683), \mathrm{n} 21$ latency $(Z=1.070, p=0.285)$, EMG normalized peak-to-peak amplitude $(Z=0.613, p=0.540)$, and mean rectified EMG $(Z=1.419, p=0.156)$. The results also showed no significant ear differences in the contralateral mVEMP's p11 latency $(Z=0.074, p=0.941), \mathrm{n} 21$ latency $(Z=1.068, p=0.285)$, EMG normalized peak-to-peak amplitude $(Z=0.069$, $p=0.945)$, and mean rectified EMG $(Z=1.794, p=0.073)$.

\section{Effect of Sex on mVEMP Parameters}

Mean, median, and standard deviation of p11 latency, n21 latency, EMG normalized peak-to-peak amplitude, mean rectified EMG, and interaural amplitude ratio of ipsilateral and contralateral tone burst mVEMP responses recorded from 12 males and 32 females are shown in - Table 1. Latency

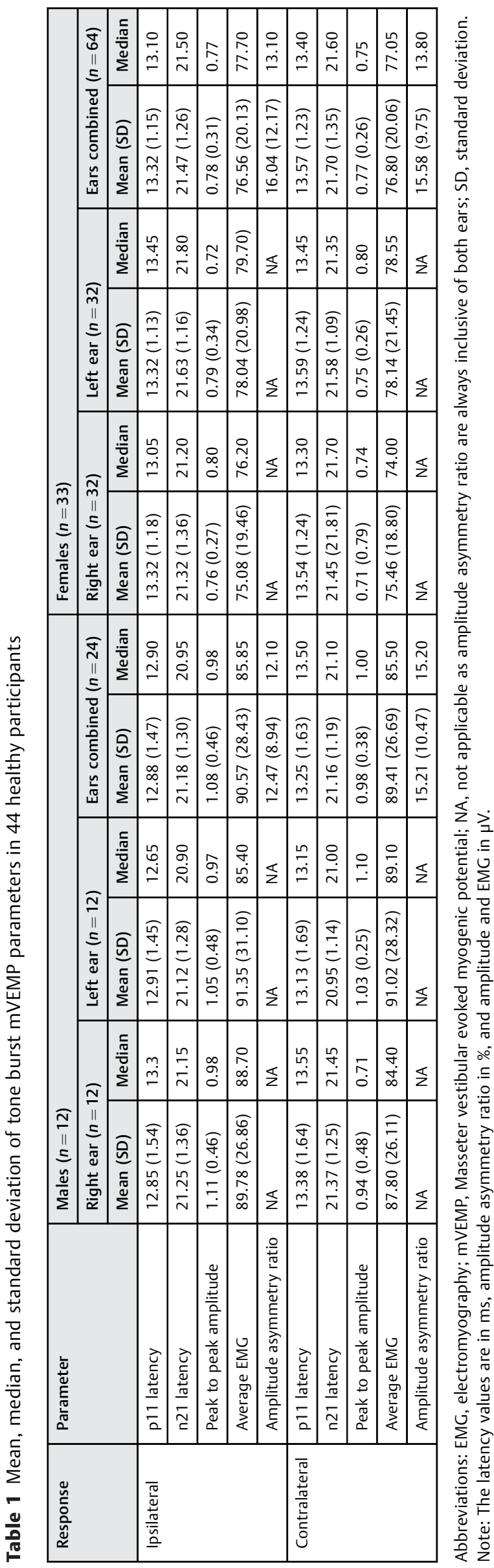


parameters (mean latencies of p11 and n21) of ipsilateral and contralateral tone burst mVEMP responses were similar in both the sex. Mann-Whitney U test showed no significant difference between males and females in ipsilateral p11 latency $(Z=1.093, \quad p=0.245)$, ipsilateral n21 latency $(Z=0.839, p=0.401)$, contralateral p11 latency $(Z=0.338$, $p=0.736)$, contralateral n21 latency $(Z=1.566, p=0.117)$, ipsilateral amplitude asymmetry ratio $(Z=0.485, p=0.490)$, and contralateral amplitude asymmetry ratio $(Z=0.947$, $p=0.948$ ). However, males had significantly larger ipsilateral EMG normalized peak-to-peak amplitude of $(Z=3.015$, $p=0.003)$, contralateral EMG normalized peak-to-peak amplitude $(Z=2.410, p=0.016)$, and ipsilateral mean rectified EMG $(Z=1.977, p=0.048)$, as can be seen in - Table 1. Although the mean rectified EMGs of contralateral mVEMP responses were higher in males than females, they were not significantly different $(Z=1.874, p=0.061)$.

\section{The Test-Retest Reliability of mVEMP}

Ten participants underwent mVEMP testing a second time within a month of being tested the first time. Mean, standard deviation, median, and ICC of various parameters of mVEMP of both sessions are shown in - Table $\mathbf{2}$. The ICC values showed excellent test-retest reliability (ICC $>0.75$ ) for ipsilateral p11 latency, contralateral p11 latency, ipsilateral EMG normalized peak-to-peak amplitude, and ipsilateral amplitude asymmetry ratio. Fair-to-good test-retest reliability (ICC values between 0.4 and 0.75 ) was observed for ipsilateral n21 latency, contralateral n21 latency, contralateral EMG normalized peak-topeak amplitude, and contralateral amplitude asymmetry ratio, as seen in - Table 2. - Fig. $\mathbf{2}$ shows grand averaged waveforms of ipsilateral and contralateral tone burst mVEMP responses from 10 healthy individuals recorded during the first and second session.

\section{Discussion}

\section{Normative Values of Tone Burst mVEMP}

This study was performed to establish normative values for tone burst mVEMP and determine its test-retest reliability. Normative values of unilateral tone burst evoked mVEMP responses showed a bilateral and symmetrical response like click-evoked mVEMP ${ }^{5}$ that can be consistently recorded on all participants. Comparison of tone burst mVEMP responses between the ears showed that these VEMP parameters are similar between both the ears without any significant difference. From the review of literature and to the best of our knowledge this is the first study on tone burst evoked mVEMP responses. Hence, the comparison was made with click-evoked mVEMP that were extensively studied by Deriu and colleagues. ${ }^{5,7,8,13,14}$

As with click mVEMP, tone burst mVEMP shows robust and clear positive peak (p11) responses followed by later variable negative peak (n21) responses. The tone burst mVEMP responses were prolonged in latency and increased amplitude when compared with click-evoked mVEMP responses. ${ }^{13}$ The mean values of ipsilateralp11, n21 latency and contralateral p11, n21 latency of tone burst mVEMP

\begin{tabular}{|c|c|c|c|c|c|c|c|c|c|c|c|}
\hline & 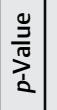 & $\begin{array}{l}\bar{\delta} \\
\stackrel{0}{0} \\
\dot{v}\end{array}$ & $\mid \begin{array}{l}\tilde{o} \\
\dot{o} \\
\dot{0}\end{array}$ & $\mid \begin{array}{c}\bar{c} \\
\dot{\sigma} \\
\bar{v}\end{array}$ & $\begin{array}{l}\bar{\alpha} \\
\bar{o} \\
\dot{0}\end{array}$ & $\begin{array}{l}\bar{b} \\
\dot{0} \\
\dot{v}\end{array}$ & $\begin{array}{l}\bar{\vdots} \\
\dot{0} \\
\dot{v}\end{array}$ & 㟔 & $\begin{array}{l}\tilde{z} \\
\delta \\
0 \\
0\end{array}$ & $\begin{array}{l} \\
\dot{0} \\
\dot{0} \\
\end{array}$ & 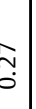 \\
\hline & $\frac{\mathbf{v}}{\mathbf{5}}$ & $g$ & $\stackrel{g}{9}$ & 9 & a & a & 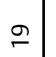 & 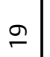 & 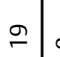 & $\sigma$ & $a$ \\
\hline & 5 & $\stackrel{9}{\neg}$ & 9 & 9 & $a$ & a & 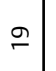 & $\stackrel{9}{-1}$ & $\stackrel{\circ}{-}$ & $\sigma$ & $\sigma$ \\
\hline & 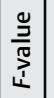 & $\underset{\substack{\tilde{N} \\
\infty}}{\hat{\infty}}$ & $\mid \begin{array}{l}q \\
o \\
o \\
m\end{array}$ & $\stackrel{\text { F }}{\rightleftharpoons}$ & $\mid \begin{array}{l}n \\
\hat{n} \\
\stackrel{0}{0}\end{array}$ & $\begin{array}{l}\stackrel{0}{\tilde{\sim}} \\
\stackrel{\sim}{*}\end{array}$ & 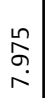 & $\begin{array}{l}\bar{\sigma} \\
\dot{m}\end{array}$ & $\begin{array}{l}g \\
\dot{q} \\
\dot{+}\end{array}$ & 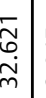 & $\begin{array}{l}q \\
\text { of } \\
m\end{array}$ \\
\hline$\stackrel{\underline{n}}{\underline{\underline{n}}}$ & & $\begin{array}{l}\frac{j}{2} \\
\frac{2}{2} \\
\end{array}$ & $\begin{array}{l}\text { ò } \\
\text { o. } \\
\text { on }\end{array}$ & $\mid$\begin{tabular}{l}
$\stackrel{\leftrightarrow}{0}$ \\
\multirow{0}{0}{}
\end{tabular} & $\mid \begin{array}{l}n \\
\hat{\alpha} \\
\text { on } \\
0\end{array}$ & $\begin{array}{c}0 \\
0 \\
0 \\
0 \\
0\end{array}$ & \begin{tabular}{l}
$n$ \\
\multirow{2}{0}{} \\
0
\end{tabular} & $\begin{array}{c}\vdots \\
\infty \\
\infty \\
\vdots \\
\vdots\end{array}$ & 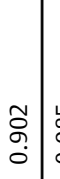 & & 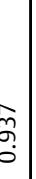 \\
\hline 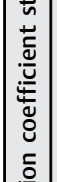 & 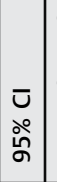 & 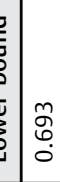 & 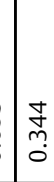 & 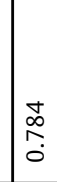 & 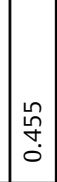 & $\begin{array}{c}\stackrel{2}{2} \\
0 \\
0 \\
0\end{array}$ & $\begin{array}{l}\tilde{0} \\
0 \\
0 \\
0\end{array}$ & 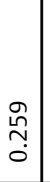 & 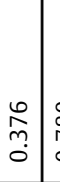 & & : \\
\hline 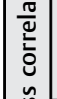 & $\underline{u}$ & 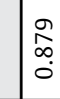 & 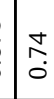 & $\begin{array}{l}n \\
\sigma \\
\sigma \\
0\end{array}$ & $\mid \begin{array}{c}0 \\
0 \\
0 \\
0 \\
0\end{array}$ & \begin{tabular}{l}
$\infty$ \\
\multirow{2}{0}{} \\
$\vdots$ \\
0
\end{tabular} & $\begin{array}{c}n \\
0 \\
\infty \\
0 \\
0\end{array}$ & $\begin{array}{l}0 \\
? \\
0\end{array}$ & $\begin{array}{c}n \\
\stackrel{n}{n} \\
\dot{0}\end{array}$ & $\begin{array}{l} \\
\text { t. } \\
\\
\end{array}$ & $\begin{array}{l}\frac{f}{d} \\
0 \\
0\end{array}$ \\
\hline 竧 & б & $\begin{array}{l}\stackrel{2}{2} \\
\infty \\
0 \\
0\end{array}$ & $\underset{0}{\stackrel{N}{0}}$ & $\begin{array}{l}n \\
\sigma \\
\sigma \\
0\end{array}$ & $\mid \begin{array}{l}\hat{0} \\
0 \\
0 \\
0\end{array}$ & 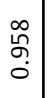 & $\begin{array}{c}n \\
0 \\
\infty \\
0 \\
0\end{array}$ & $\begin{array}{l}0 \\
0 \\
0\end{array}$ & 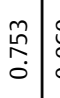 & 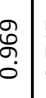 & $\begin{array}{l}\frac{f}{d} \\
0 \\
0\end{array}$ \\
\hline & 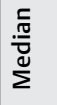 & 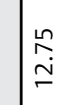 & $\bar{v}$ & $\stackrel{\infty}{\stackrel{\infty}{-}}$ & $\mid \begin{array}{l}n \\
\tilde{m} \\
\dot{\sigma} \\
\infty\end{array}$ & 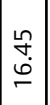 & $\begin{array}{l}\stackrel{n}{\mathfrak{i}} \\
\stackrel{\sim}{\sim}\end{array}$ & 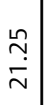 & $\stackrel{n}{\longrightarrow}$ & 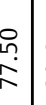 & $\begin{array}{l}\text { : } \\
\text { i. }\end{array}$ \\
\hline & î & 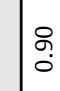 & $\stackrel{\circ}{\circ}$ & ¿ & $\begin{array}{l}\stackrel{ \pm}{m} \\
\stackrel{\sim}{ \pm}\end{array}$ & 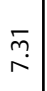 & $\begin{array}{l}\hat{\infty} \\
0 \\
0\end{array} \mid$ & $\stackrel{\simeq}{\check{C}}$ & $\begin{array}{c}\stackrel{m}{m} \\
\dot{m}\end{array}$ & $\begin{array}{l}\bar{\sigma} \\
\dot{ \pm} \\
\end{array}$ & 我 \\
\hline 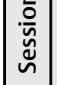 & $\begin{array}{l}\text { 乓 } \\
\sum^{\mathbb{N}}\end{array}$ & 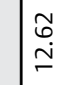 & $\frac{\dot{\alpha}}{\dot{\sim}}$ & 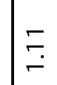 & $\mid \begin{array}{c}\tilde{m} \\
\stackrel{m}{\infty} \\
\dot{\infty}\end{array}$ & $\begin{array}{l}\hat{N} \\
\stackrel{6}{0}\end{array}$ & 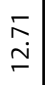 & $\begin{array}{l}\stackrel{u}{0} \\
\dot{\sim}\end{array}$ & $\underset{\dot{r}}{\dot{\sim}}$ & $\begin{array}{l}\underset{\sim}{\infty} \\
\stackrel{\infty}{\circ}\end{array}$ & $\begin{array}{l}\underbrace{}_{0} \\
\dot{\omega} \\
\underline{\omega}\end{array}$ \\
\hline & 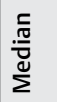 & $\stackrel{\sim}{\underset{\Upsilon}{\sim}}$ & $\begin{array}{l}n \\
\alpha \\
\grave{N}\end{array}$ & $\underset{r}{\stackrel{\circ}{\circ}}$ & 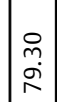 & $\mid \begin{array}{l}0 \\
\infty \\
\dot{\varphi} \\
-\end{array}$ & $\begin{array}{l}\stackrel{P}{i} \\
\stackrel{1}{i}\end{array}$ & $\stackrel{n}{\grave{N}}$ & 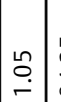 & 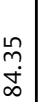 & $\frac{n}{6}$ \\
\hline & in & $\stackrel{\leftrightarrow}{\stackrel{\leftrightarrow}{+}}$ & $\underset{F}{F}$ & $\underset{-}{\stackrel{-}{2}}$ & 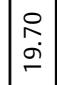 & 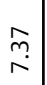 & $\stackrel{0}{-}$ & $\stackrel{\tilde{m}}{\sim}$ & $\begin{array}{c}\hat{m} \\
0\end{array}$ & 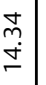 & f. \\
\hline 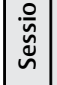 & 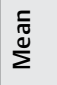 & $\stackrel{\stackrel{n}{N}}{\underset{N}{N}}$ & $\stackrel{\stackrel{\rho}{\sim}}{\stackrel{\sim}{\sim}}$ & 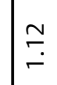 & 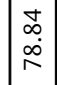 & 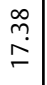 & $\begin{array}{l}\stackrel{\sim}{\infty} \\
\stackrel{\sim}{\simeq}\end{array}$ & 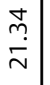 & $\stackrel{\circ}{\stackrel{0}{0}}$ & 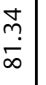 & $\begin{array}{l}\text { D. } \\
\text { m. } \\
c\end{array}$ \\
\hline 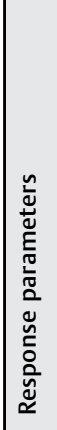 & & 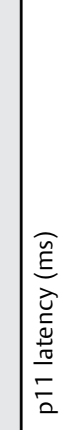 & 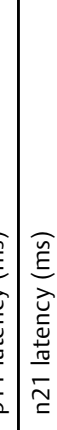 & 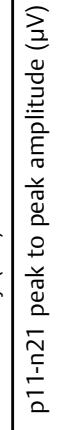 & 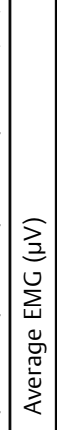 & 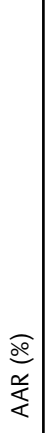 & 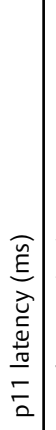 & 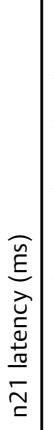 & 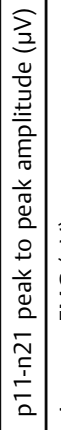 & 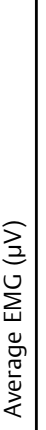 & $\begin{array}{l}\frac{0}{4} \\
\frac{\alpha}{4}\end{array}$ \\
\hline 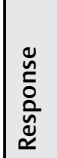 & & 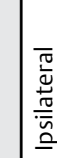 & & & & & 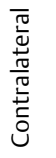 & & & & \\
\hline
\end{tabular}


312 Tone Burst Masseter Vestibular Evoked Myogenic Potentials Vignesh et al.

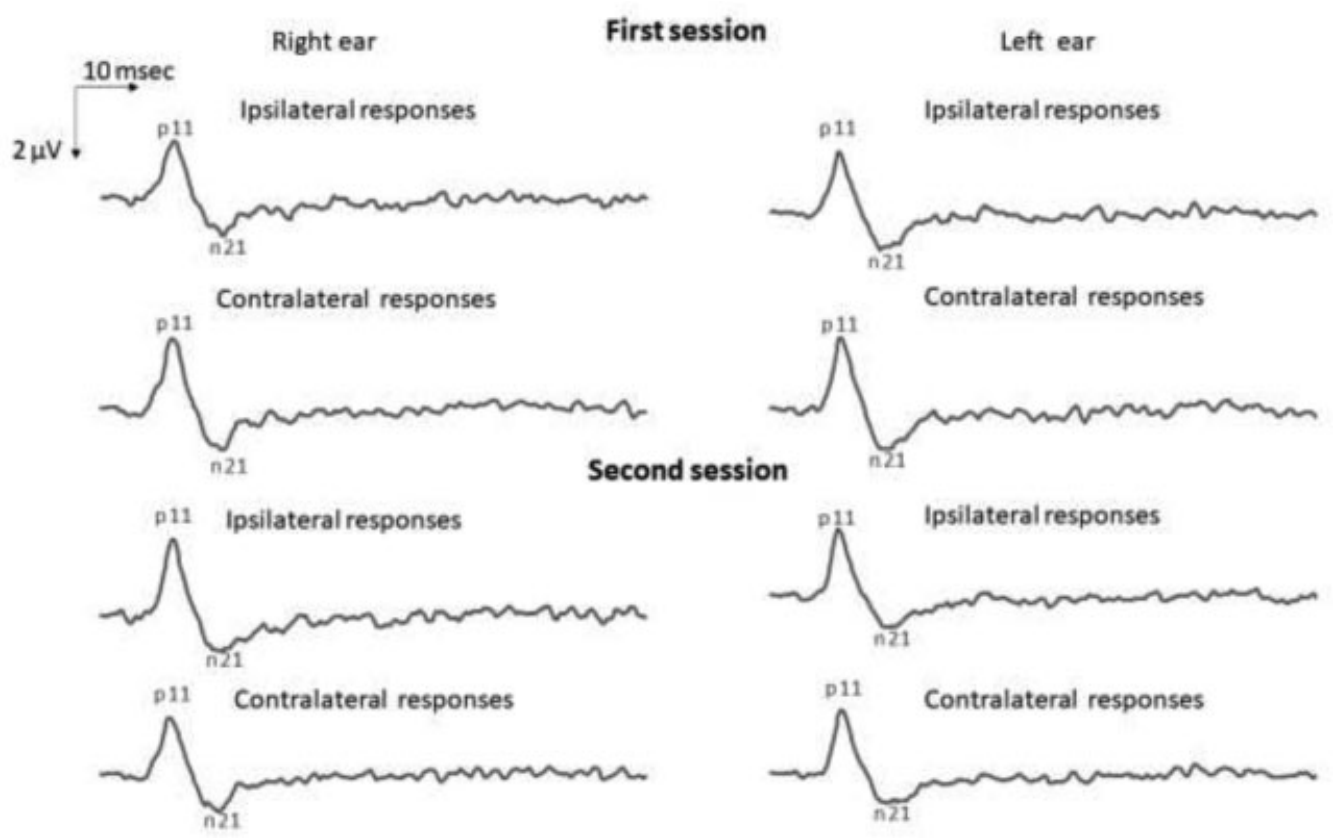

Fig. 2 Grand averaged waveforms of ipsilateral and contralateral tone burst mVEMP responses from 10 healthy participants during the first and second session of testing. mVEMP, masseter vestibular evoked myogenic potential.

were prolonged in the present study when compared with corresponding values of the click mVEMP responses reported by De Natale et al. ${ }^{13}$ Similarly, the mean EMG normalized peak-to-peak amplitudes of ipsilateral and contralateral tone burst mVEMP responses were higher in tone burst mVEMP responses when compared with click-evoked mVEMP responses as evidenced in - Table 3 . These EMG normalized peak-to-peak amplitudes of tone burst mVEMP were higher even with the lower intensity level of stimulation (125 peSPL) when compared with click-evoked mVEMP ${ }^{13}$ at $138 \mathrm{~dB}$ peSPL. The amplitude asymmetry ratio of ipsilateral and contralateral responses was similar to the corrected ampli- tude asymmetry ratio of click-evoked responses reported by De Natale et al. ${ }^{13}$ These observations are similar to the findings in the cervical ${ }^{15,23-25}$ and oVEMP ${ }^{16}$ that report increased latency and higher amplitude for tone burst stimuli than click stimuli.

\section{Effect of Sex on mVEMP Parameters}

Normative studies on click-evoked mVEMP responses ${ }^{13,14}$ have reported significant differences inp11 and n21 latencies between males and females. Females were reported to show shorter latencies of click-evoked mVEMP responses than males. These differences were attributed to the difference

Table 3 Comparison of tone burst mVEMP findings in this study with the click evoked mVEMP findings published earlier

\begin{tabular}{|c|c|c|c|}
\hline \multirow[t]{3}{*}{ Response } & \multirow[t]{3}{*}{ Parameter } & $\begin{array}{l}\text { Tone burst evoked mVEMP } \\
\text { Present study }\end{array}$ & $\begin{array}{l}\text { Click evoked mVEMP } \\
\text { De Natale et al }{ }^{13}\end{array}$ \\
\hline & & Total $(n=44)$ & Total $(n=62)$ \\
\hline & & Mean (SD) & Mean (SD) \\
\hline \multirow[t]{5}{*}{ Ipsilateral } & p11 latency & $13.20(1.25)$ & $11.17(0.98)$ \\
\hline & n21 latency & $21.40(1.27)$ & $19.68(1.81)$ \\
\hline & Peak to peak amplitude & $0.86(0.38)$ & $0.72(0.31)$ \\
\hline & Average EMG & $80.38(23.38)$ & $104.31(41.66)$ \\
\hline & Amplitude asymmetry ratio & $15.07(11.40)$ & $14.56(11.8)$ \\
\hline \multirow[t]{5}{*}{ Contralateral } & p11 latency & $13.48(1.35)$ & $11.38(0.9)$ \\
\hline & n21 latency & $21.55(1.32)$ & $19.53(1.9)$ \\
\hline & Peak to peak amplitude & $0.83(0.31)$ & $0.74(0.31)$ \\
\hline & Average EMG & $80.24(22.62)$ & $102(40.96)$ \\
\hline & Amplitude asymmetry ratio & $15.48(9.83)$ & Not measured \\
\hline
\end{tabular}

Abbreviations: EMG, electromyography; mVEMP, Masseter vestibular evoked myogenic potentials; SD, standard deviation.

Note: The latency values are in ms, amplitude asymmetry ratio in \%, and amplitude and EMG in $\mu \mathrm{V}$. 
in the cochlear average length, which is lower in females. ${ }^{13}$ Though the sex differences are not observed in the cervical ${ }^{26}$ and oVEMP, ${ }^{27}$ the sex difference in MVEMP due to cochlear anatomy is questionable. ${ }^{28}$ The present study showed no significant difference in $\mathrm{p} 11$ and $\mathrm{n} 21$ latencies between the sex, instead, we observed significant differences in EMG normalized peak-to-peak amplitude between the males and females. These sex differences in EMG normalized peak-to-peak amplitude were not reported by any other research studies using click-evoked mVEMP.

In this present study, the mean EMG normalized peak to peak amplitudes of ipsilateral $(1.08 \mu \mathrm{V})$ and contralateral p11$\mathrm{n} 21(0.98 \mu \mathrm{V})$ were higher in males than amplitudes of ipsilateral $(0.78 \mu \mathrm{V})$ and contralateral p11-n21 $(0.77 \mu \mathrm{V})$ of females. Sex difference in the present study could be due to: (1) higher mean rectified EMG values in males than females observed in this study; (2) masseter muscle is thicker in males than females; ${ }^{29}$ (3) volume of the trigeminal nerve is larger in males than females ${ }^{30}$ and (4) difference in the number of male $(n=12)$ and female $(n=32)$ participants considered in this study. The mean maximum voluntary contraction observed in males $(210.75 \mu \mathrm{V})$ was higher in males than females (168.72 $\mu \mathrm{V})$. Since the EMG target levels for recording mVEMP were set to 30 to $50 \%$ of maximum voluntary contraction, it would have led to increased EMG target levels in males than females. Though EMG normalization was used for mVEMP, as used in cVEMPs, in this study to reduce the effects of the increase in amplitude with increased muscle tension levels, ${ }^{31}$ we observed a significant difference in amplitude levels between males and females in mVEMP recordings. Hence other factors of the thicker masseter muscle and increased volume of the trigeminal nerve may play a role in increased amplitude of tone burst mVEMP in males. Similar findings of higher oVEMP amplitude in males than females are reported in oVEMP studies. ${ }^{27,32}$ These differences were attributed to larger muscle bulk of inferior oblique muscles in males than females. ${ }^{27}$

\section{Test-Retest Reliability of mVEMP}

Tone burst mVEMP in the present study showed excellent testretest reliability, which is an essential indicator for any test to use in clinical practice. Though there are no studies investigating the test-retest reliability of even click-evoked mVEMP this study highlights that tone burst mVEMP has high reliability in healthy individuals. The positive $\mathrm{p} 11$ peak of tone burst mVEMP in this study has excellent test-retest reliability which is described as a clear and well-defined response in click-evoked mVEMP responses. ${ }^{8}$ But, later negative peak n21 showed fair test-retest reliability which is described as a variable response in clickevoked mVEMP by Deriu et al. ${ }^{8}$ Though several parameters showed excellent and fair test-retest reliability, there was individual variation between participants, which could be due to variation in the amount of masseter muscle activation between participants. Also, we observed variation in tone burst mVEMP responses on some participants between first and sessions of recording possible due to variations in electrode placement between the sessions.

mVEMP recording can therefore be a simple, inexpensive, fast, and reliable test that is well tolerated and can be easily implemented in any laboratory that has cVEMP recording facilities or evoked potentials systems. Because mVEMP is a vestibular evoked potential, sharing pathway that is common with cervical and oVEMP, ${ }^{9}$ it would be interesting to check its clinical application in various peripheral and central vestibular disorders. Click evoked mVEMPs have been studied in few clinical populations including conductive hearing loss, ${ }^{5}$ profound sensory neural hearing loss, vestibular neuritis, complete removal of the auditory and vestibular nerve (surgical excision of acoustic schwannoma), ${ }^{8}$ Parkinson's disease, ${ }^{6,12}$ and multiple sclerosis. ${ }^{10,11}$ Especially mVEMP abnormalities are reported to be higher than cVEMP (in multiple sclerosis), cVEMP and oVEMP (in Parkinson's disease) to identifying and monitoring brainstem dysfunction. $^{10-12}$ Therefore, in addition to cervical and oVEMP, mVEMP can be useful in assessing vestibulomasseteric pathways along the brainstem. Also, tone burst mVEMPs are larger in amplitude when compared with click-evoked mVEMP, hence tone burst stimuli can be of choice in recording mVEMP. Though this potential is robust and reliable it lacks investigation of studies like the origin of this potential in vestibular apparatus, normative in a large population with different age groups, and frequency tuning of mVEMP, for its clinical use. Though we provide normative data for tone burst mVEMP, the number of participants in this study is small and could be a drawback for this study. More research is needed with a larger sample size to be absolutely certain of these normative values.

\section{Conclusion}

The present study was carried to establish normative data for tone burst mVEMP and determine its test-retest reliability. It was observed that tone burst mVEMP responses are robust and consistently observed on all participants with prolonged latencies and higher amplitude over click-evoked mVEMP responses. The latencies of tone burst mVEMP responses were similar across males and females but the amplitudes were higher in males than females. Also, this test showed excellent or fair test-retest reliability on all participants. Therefore, mVEMP can be a useful tool in evaluating vestibular and trigeminal pathway in various peripheral and central vestibular disorders.

Conflict of Interest

None declared.

\section{References}

1 Rosengren SM, Colebatch JG, Young AS, Govender S, Welgampola MS. Vestibular evoked myogenic potentials in practice: methods, pitfalls and clinical applications. Clin Neurophysiol Pract 2019;4:47-68

2 Rudisill HE, Hain TC. Lower extremity myogenic potentials evoked by acoustic stimuli in healthy adults. Otol Neurotol 2008;29(05): 688-692

3 Cherchi M, Bellinaso NP, Card K, et al. Sound evoked triceps myogenic potentials. Otol Neurotol 2009;30(04):545-550

4 Ferber-Viart C, Soulier N, Dubreuil C, Duclaux R. Cochleovestibular afferent pathways of trapezius muscle responses to clicks in human. Acta Otolaryngol 1998;118(01):6-10 
5 Deriu F, Tolu E, Rothwell JC. A sound-evoked vestibulomasseteric reflex in healthy humans. J Neurophysiol 2005;93(05):2739-2751

6 de Natale ER, Ginatempo F, Paulus KS, et al. Paired neurophysiological and clinical study of the brainstem at different stages of Parkinson's disease. Clin Neurophysiol 2015;126(10):1871-1878

7 Deriu F, Tolu E, Rothwell JC. A short latency vestibulomasseteric reflex evoked by electrical stimulation over the mastoid in healthy humans. J Physiol 2003;553(Pt 1):267-279

8 Deriu F, Ortu E, Capobianco S, et al. Origin of sound-evoked EMG responses in human masseter muscles. J Physiol 2007;580 (Pt 1):195-209

9 Deriu F, Giaconi E, Rothwell JC, Tolu E. Reflex responses of masseter muscles to sound. Clin Neurophysiol 2010;121(10):1690-1699

10 Magnano I, Pes GM, Pilurzi G, et al. Exploring brainstem function in multiple sclerosis by combining brainstem reflexes, evoked potentials, clinical and MRI investigations. Clin Neurophysiol 2014;125(11):2286-2296

11 Magnano I, Pes GM, Cabboi MP, et al. Comparison of brainstem reflex recordings and evoked potentials with clinical and MRI data to assess brainstem dysfunction in multiple sclerosis: a shortterm follow-up. Neurol Sci 2016;37(09):1457-1465

12 de Natale ER, Ginatempo F, Paulus KS, et al. Abnormalities of vestibular-evoked myogenic potentials in idiopathic Parkinson's disease are associated with clinical evidence of brainstem involvement. Neurol Sci 2015;36(06):995-1001

13 De Natale ER, Ginatempo F, Mercante B, et al. Vestibulo masseteric reflex and acoustic masseteric Reflex. Normative data and effects of age and gender. Clin Neurophysiol 2019;130(09):1511-1519

14 Ginatempo F, Ortu E, Pilurzi G, Tolu E, Deriu F. Vestibulo-masseteric reflex (VMR) and acoustic-masseteric reflex (AMR): normative values. Clin Neurophysiol 2013;124:216

15 Akin FW, Murnane OD, Proffitt TM. The effects of click and toneburst stimulus parameters on the vestibular evoked myogenic potential (VEMP). J Am Acad Audiol 2003;14(09):500-509, quiz 534-535

16 Deepak DT, Bhat JS, Kumar K. Ocular vestibular evoked myogenic potential using different test stimuli. ISRN Otolaryngol 2013; 2013:161937

17 Murnane OD, Akin FW, Kelly KJ, Byrd S. Effects of stimulus and recording parameters on the air conduction ocular vestibular evoked myogenic potential. J Am Acad Audiol 2011;22(07): 469-480

18 Welgampola MS, Colebatch JG. Characteristics of tone burstevoked myogenic potentials in the sternocleidomastoid muscles. Otol Neurotol 2001;22(06):796-802
19 Park HJ, Lee I-S, Shin JE, Lee YJ, Park MS. Frequency-tuning characteristics of cervical and ocular vestibular evoked myogenic potentials induced by air-conducted tone bursts. Clin Neurophysiol 2010;121(01):85-89

20 Singh NK, Keloth NK, Sinha S. Is there a safe level for recording vestibular evoked myogenic potential? Evidence from cochlear and hearing function tests. Ear Hear 2019;40(03):493-500

21 Welgampola MS, Colebatch JG. Vestibulocollic reflexes: normal values and the effect of age. Clin Neurophysiol 2001;112(11): 1971-1979

22 Versino M, Colnaghi S, Callieco R, Cosi V. Vestibular evoked myogenic potentials: test-retest reliability. Funct Neurol 2001; 16(04):299-309

23 Kumar K, Sinha SK, Bharti AK, Barman A. Comparison of vestibular evoked myogenic potentials elicited by click and short duration tone burst stimuli. J Laryngol Otol 2011;125(04):343-347

24 Özgür A, Çelebi Erdivanlı Ö, Özergin Coşkun Z, et al. Comparison of tone burst, click and chirp stimulation in vestibular evoked myogenic potential testing in healthy people. J Int Adv Otol 2015;11(01):33-35

$25 \mathrm{Wu}$ H-J, Shiao A-S, Yang Y-L, Lee G-S. Comparison of short tone burst-evoked and click-evoked vestibular myogenic potentials in healthy individuals. J Chin Med Assoc 2007;70(04):159-163

26 Ochi K, Ohashi T. Age-related changes in the vestibular-evoked myogenic potentials. Otolaryngol Head Neck Surg 2003;129(06): 655-659

27 Sung P-H, Cheng P-W, Young Y-H. Effect of gender on ocular vestibular-evoked myogenic potentials via various stimulation modes. Clin Neurophysiol 2011;122(01):183-187

28 Miller JD. Sex differences in the length of the organ of Corti in humans. J Acoust Soc Am 2007;121(04):EL151-EL155

29 Raadsheer MC, Kiliaridis S, Van Eijden TMGJ, Van Ginkel FC, PrahlAndersen B. Masseter muscle thickness in growing individuals and its relation to facial morphology. Arch Oral Biol 1996;41(04): 323-332

30 Kress B, Schindler M, Rasche D. Why do advanced age and female gender predispose to trigeminal neuralgia? Clin Neuroradiol 2008;18:60-64

31 McCaslin DL, Fowler A, Jacobson GP. Amplitude normalization reduces cervical vestibular evoked myogenic potential (cVEMP) amplitude asymmetries in normal subjects: proof of concept. J Am Acad Audiol 2014;25(03):268-277

32 Xie S-J, Xu Y, Bi H-Z, Jia H-B, Zheng Y-J, Zhang Y-G. Ocular vestibular-evoked myogenic potentials in healthy pilots and student pilots. Aviat Space Environ Med 2011;82(07):729-733 